\title{
Two Dimensional Analysis of Burden Flow in Blast Furnace Based on Plasticity Theory
}

\author{
Hiroshi TAKAHASHI,1) Kousei KUSHIMA ${ }^{2)}$ and Takao TAKEUCHI1)
}

1) Department of Chemical Engineering, Muroran Institute of Technology, Mizumoto-cho, Muroran, Hokkaido, 050 Japan.

2) Hirohata Works, Nippon Steel Corporation, Fuji-cho, Hirohata-ku, Himeji, Hyogo-ken, 671-11 Japan.

(Received on May 2, 1988; accepted in the final form on September 9, 1988)

\begin{abstract}
Using a two-dimensional cold model, the dynamic behavior of particles in blast furnace was studied on a detailed analysis of the flow pattern. The field of the flow was characterized with the stagnant region formed in the central lower part of the apparatus and the flow channels consisting of two regions, i.e., slow moving and fast moving regions. On crossing the boundary between the slow and fast moving regions, the particles experienced a discontinuous, sudden change in the velocity as well as the direction of flow. An approximate method of predicting the flow pattern is described on the basis of plasticity theory. The velocity discontinuous boundary could be theoretically represented by the characteristic curve of the velocity. The boundary of the stagnant region corresponded to the characteristic curve of the stress, so-called slip line. Finally, the flow pattern of burden material in a practical blast furnace was simulated, based on the calculas presented.
\end{abstract}

KEY WORDS: moving bed; blast furnace process; burden materials; flow pattern; solid pressure; slip line; dead zone; velocity discontinuity; ironmaking.

\section{Introduction}

An important requirement in the design and operation of moving-bed reactors is to be able to predict the dynamics of solid particles. Some troubles in the operation of iron making blast furnace may be caused by unstable movement of burden materials due to local growth of the stagnant region or stoppage of the solid motion. Securing the stable descent of the particles is required for a high reactor efficiency. Therefore, the motion of the particles flowing down through blast furnace has been investigated ${ }^{1-9)}$ by measuring the flow pattern of the particles and the internal stresses acting in the particles or by the theoretical consideration of the motion. The observation of the flow pattern of particles in a vessel with single aperture, revealed that the flow channel converging toward the discharge port consisted of at least two regions with different velocity characteristics, i.e., slow moving and fast moving regions. ${ }^{10)}$ The velocity field of particles set up during flowing in blast furnace may be also accompanied by such regions with different velocity characteristics. However, nothing has been reported on this subject. The present paper describes the results of an investigation on the flow pattern of the particles flowing down through a twodimensional cold model of blast furnace. The existence of the position at which the velocity and the direction of flow changed discontinuously, was confirmed experimentally. The position was theoretically represented by the characteristic curve of the velocity. Based on plasticity theory, a method of predicting the characteristic curve and the boundary of the stagnant region formed in the central lower part of the apparatus was described.

\section{Experiment}

\subsection{Experimental Apparatus and Procedure}

A two-dimensional cold model of blast furnace shown in Fig. 1 was used. The sides consisted of iron sheets, while the front and back consisted of parallel, transparent, acrylic sheets. The particles used were sand and glass beads with a uniform diameter, and the physical properties are shown in Table 1 . The angle of internal friction, $\phi$, was estimated referring to the discharge angle shown below. The angle of wall friction, $\phi_{w}$, was measured by the method described in the previous report. ${ }^{11)}$ The values of void fraction $\varepsilon$ are in the state of natural packing of the particles. The air, supplied at the bottom of the dead reagion of the particles, ascended through the apparatus and was exhausted from the top of the bed to the atomosphere. The solid particles were continuously fed from a feed hopper in such a way as to maintain a constant height at the top of the bed. The particles were fed at the center or the sides of the bed. An electromagnetic feeder situated below the outlet was used to achieve a controlled discharge of particles from the apparatus. The superficial velocity of air, $u_{f}$, and linear velocity of solid, $u_{s}$, were as follows: $u_{f}=0,0.35$ and $0.48 \mathrm{~m} / \mathrm{s}$, and $u_{s}=3.33 \times$ $10^{-4} \mathrm{~m} / \mathrm{s}$, at the top of the apparatus. Under these conditions, the following experiments were carried out. 


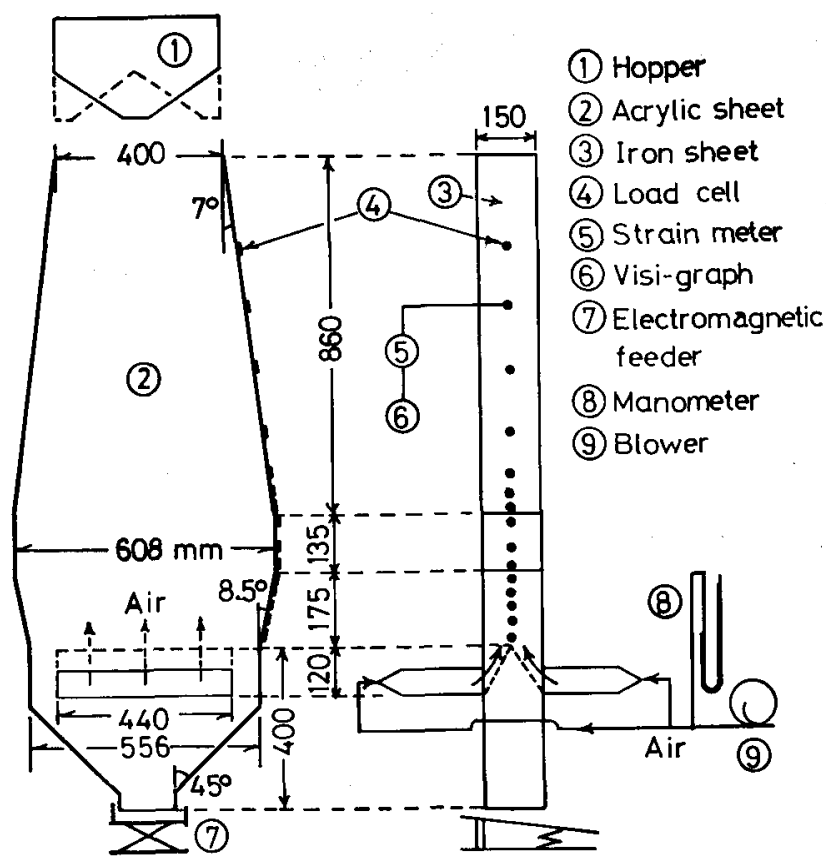

Fig. 1. Experimental apparatus.

Table 1. Physical properties of particles.

\begin{tabular}{lccccc}
\hline Material & $\begin{array}{c}d_{p} \\
\left(10^{-2} \mathrm{~m}\right)\end{array}$ & $\begin{array}{c}\rho_{p} \\
\left(\mathrm{~kg} / \mathrm{m}^{3}\right)\end{array}$ & $\begin{array}{c}\Sigma \\
(-)\end{array}$ & $\begin{array}{c}\phi \\
\left({ }^{\circ}\right)\end{array}$ & $\begin{array}{c}\phi_{w} \\
\left({ }^{\circ}\right)\end{array}$ \\
\hline Sand & 0.19 & 2610 & 0.48 & $45^{*}$ & 25 \\
Glass beads & 0.22 & 2500 & 0.39 & $35^{*}$ & 15 \\
\hline
\end{tabular}

* The value assumed referring to the angle of discharge $\phi_{d}$, defined in Fig. 9.

(1) The particles were packed naturally to form horizontal, cross-sectionally uniform layers. Layers of white-colored tracer particles (say first tracers) alternated with layers of non-colored particles. After being packed, the particles were allowed to discharge and the air was supplied at the same time. The motions of the tracer particles through the apparatus were photographed at fixed intervals of time. The boundary between the stagnant and flowing regions in the steady flow was determined and the characteristic of the flowing region was analyzed.

(2) After Experiment (1) was carried out, the discharge and feeding of particles were stopped in order to form again horizontal layers of white-colored tracers (say second tracers) at the upper region of the bed, and the flow patterns of the second tracer particles were photographed. The region of discontinuous change in the velocity, i.e., the boundary between the slow moving and fast moving regions, was determined from the points at which there were a sudden change in the deformation of second tracers.

(3) For glass beads, the distributions of the velocity and the direction of flow in the horizontal direction were measured at fixed heights of the bed by reading off the moved direction and distance of a tracer particle during a given time. The measured velocitics did not always represent the situation in the interior of the bed on account of the wall effect of the layer of one or two particles next to the wall. How- ever, in the present work, the inherent characteristics in the velocity distribution were assumed to be the same as ones in the interior of the bed. The points of discontinuous change in the velocity were found from this experiment.

(4) The dynamic pressure of the particles, acting on the side wall, was measured during flowing by means of a system consisting of load cell having diameter of $10 \mathrm{~cm}$, strain meter and visi-graph, in order to study a relationship between the flow pattern of the particles and the wall-pressure distribution.

\subsection{Experimental Results and Discussion}

The dynamic behavior in the steady flow of the particles was not varied with the method whether the particles were fed at the center or at the side walls at the top of the bed. The discription below is in the case of the central feeding. Fig. 2 shows the change of the flow pattern of sand with passage of time under the condition $u_{f}=0$ (no air flow). Only the righthand side of the flow pattern is shown because of the symmetry of the flow. As particles started to discharge, the flow region stretched upwards from the discharge ports in such a way as to form a stagnant core region in the central part of the bed, as shown in Fig. 2(a). With stretching of flow region, the stagnant region decreased in size gradually with time to reach a certain shape. The flow attained a steady state 50 to $60 \mathrm{~min}$ after the start of the discharge. When the flow attained a steady state, there appeared a very slow moving region next to fast moving region, recognized by a wavelike pattern of tracer layers near the top of the stagnant region, as shown in Figs. 2(b) and 2(c). The boundary between these two regions was discernible as the points at which the deformation of tracer particles suddenly changed. Therefore, such a boundary may correspond to the theoretical characteristic line of the velocity, at which the particles experience discontinuous change in both the velocity as well as the direction of flow. ${ }^{11,12)}$ The particles in the fast moving region descended with a velocity about 5 to 6 times faster than that in the slow moving region at the middle height of the belly section.

Figs. 2(d) and 2(e) show the patterns of second tracers, obtained from Experiment (2). A sudden change in the deformation of the tracer particles was observed, showing the presence of two regions with different velocity characteristics. The data on the points of sudden change in the pattern of second tracers, the outer contour of slow moving region and the boundary of the stagnant region in the steady state flow are plotted together in Fig. 3(a) for the case $u_{f}=0$ and in Fig. $3(\mathrm{~b})$ for the case $u_{f}=0.35 \mathrm{~m} / \mathrm{s}$. The figures show apparently that the position of severe deformation of the second tracers is a satisfactory representation for the boundary between the slow moving and fast moving regions. Consequently, the flow pattern could be divided into stagnant region and two flow regions with different velocity characteristics except for the region next to the wall where a thin, slow moving layer was formed due to the wall effect, as shown in Fig. 2. It was suggested from comparison 

(a) $t=12 \mathrm{~min}$
(b) $t=48 \mathrm{~min}$
(c) $t=54 \mathrm{~min}$
(d) $t=132 \mathrm{~min}$
(e) $t=144 \mathrm{~min}$

Fig. 2. Change of flow pattern of sand with time.

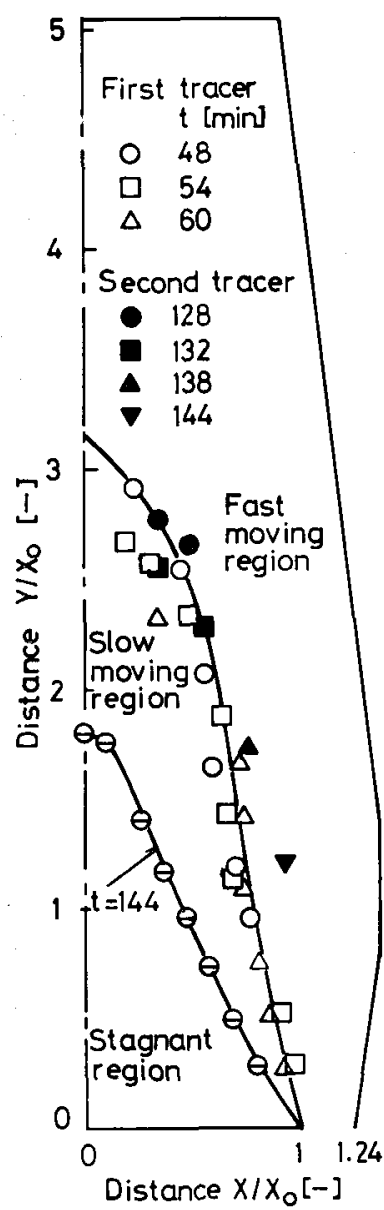

(a) $u_{f}=0$

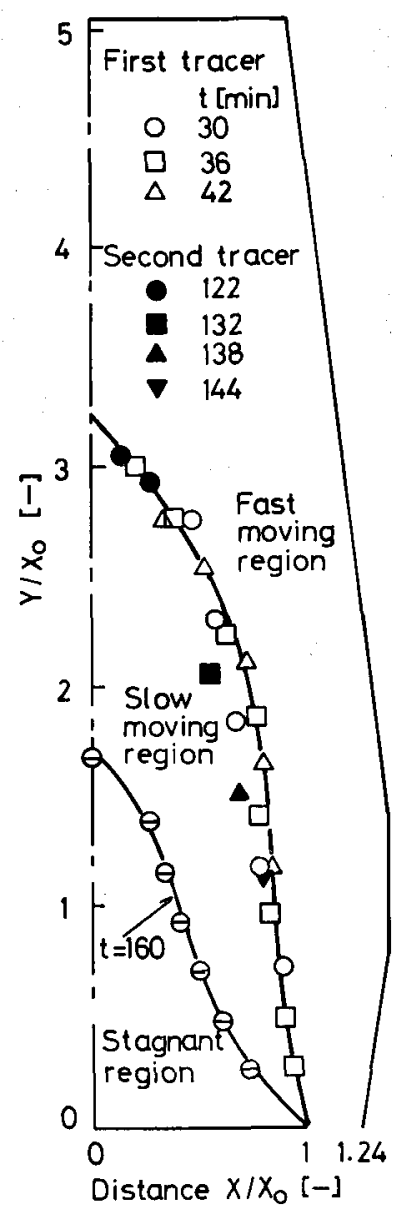

(b) $u_{f}=0.35 \mathrm{~m} / \mathrm{s}$
Fig. 3. Three characteristic regions found in the steady state flow of sand.

of Fig. 3(a) with Fig. 3(b) that the effect of flow rate of air on the flow pattern was negligible small. The motion of the burden materials consisting of ore and coke is considered to be affected by gas velocity. ${ }^{6,9}$ ) K. Narita et $a l .{ }^{6}$ ) found the effect when the gas velocity was faster than $0.8 \mathrm{~m} / \mathrm{s}$. This value seems larger than the minimum fluidization velocity of coke

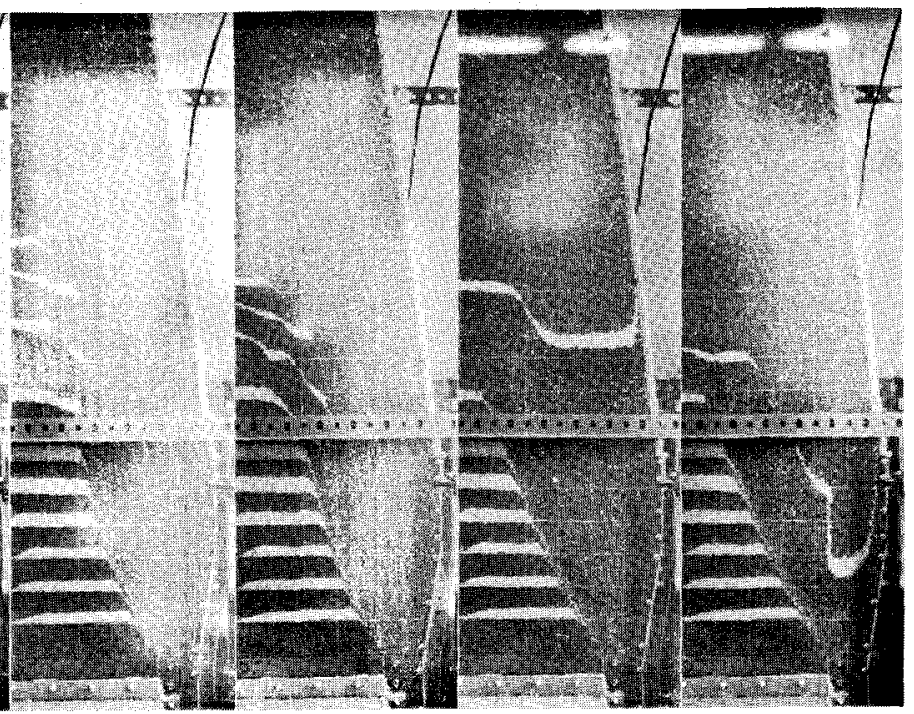

(b)

(c)

(d)

(e)

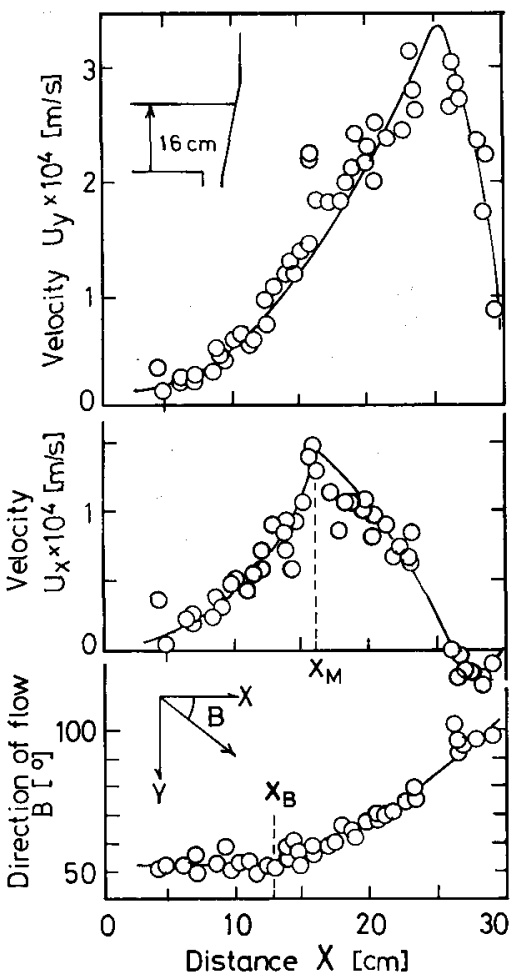

Fig. 4. Distribution of the velocity and direction of flow of glass beads.

particles used in their experiment. No effect of gas velocity found in Fig. 3 can be attributed to that the gas velocity was smaller than the minimum fluidization velocity of sand used $(\simeq 0.75 \mathrm{~m} / \mathrm{s})$, so that the friction properties of the bed were not significantly affected by gas flow.

Fig. 4 shows the experimental results in the form of the distributions of $U_{y}$ (axial velocity component), $U_{x}$ (horizontal velocity component) and $B$ (direction of flow) at the height indicated in the figure. The followings are apparent from the figure.

(1) $U_{x}$ was a maximum at a certain distance $x=$ $x_{M}$ from the central axis.

(2) The value of $U_{x}$ dropped with increasing dis- 
tance in the region $x>x_{M}$, while $U_{y}$ still increased to a maximum.

(3) The value of $B$ began to increase from a distance $x=x_{B}$ with increasing distance. These results prove that the velocity changes rapidly or discontinuously at a position being close to $x_{B}$ or $x_{M}$. The value of $B=90^{\circ}$ means the vertical descent of particles. Therefore, the negative value of $U_{x}$ and the value of $B$ larger than $90^{\circ}$ indicate simply that the situation is in the converging flow with a maximum of $U_{y}$ in the bosh section. In the flow of glass beads, the wavelike pattern of the first tracers as found in the flow of sand was not observed. The inflection point observed on the pattern of tracer with a smooth curve, instead of the points of sudden change in the flow pattern, was only information on the characteristic of the velocity field which could be obtained from Experiments (1) and (2). Such a difference between the bchaviors of sand and glass beads may be due to the difference in their friction properties. Fig. 5 shows the distributions of these inflection points and the characteristic points $x_{M}$ and $x_{B}$ in the entire flow region. The flow pattern could be described by 3 regions and conformed to the same classification as in the flow of sand.

Figs. 6 and 7 show the distribution of the dynamic wall-pressure of the particles in the steady state flow. The pressure detected on the load cell was recorded with considerable fluctuation with time, as reported by Shimizu et al." $\quad$ The plotted values are the timesmoothed ones. The two characteristic curves obtained experimentally, one related to the outer contour of the stagnant region and one related to the velocity discontinuous line, are also shown in the figures. The wall pressure was a maximum at the slightly upper height from the top of the stagnant region. The bed of particles, which moved down vertically, is being splitted into two flow regions near the level. The horizontal component of the normal stress may vary to become greater than the vertical

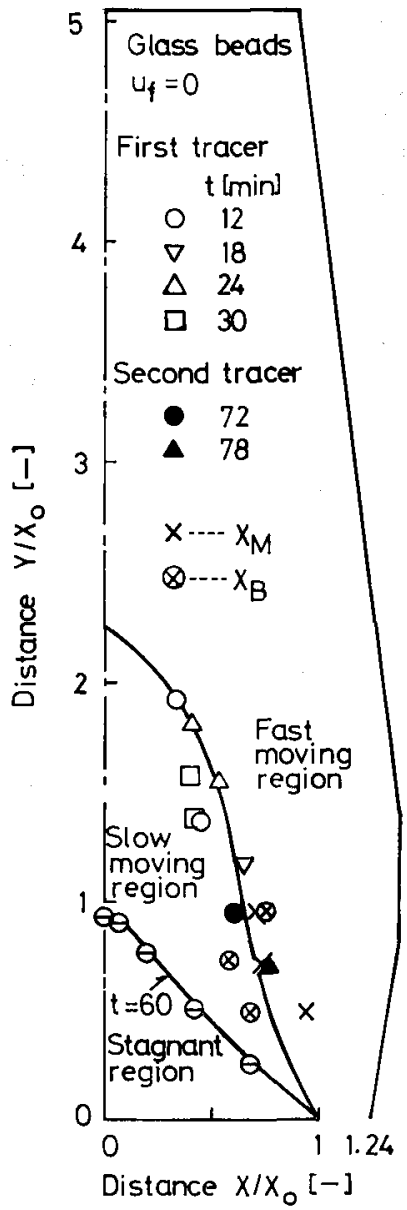

Fig. 5.

Three characteristic regions found in the steady state flow of glass beads.

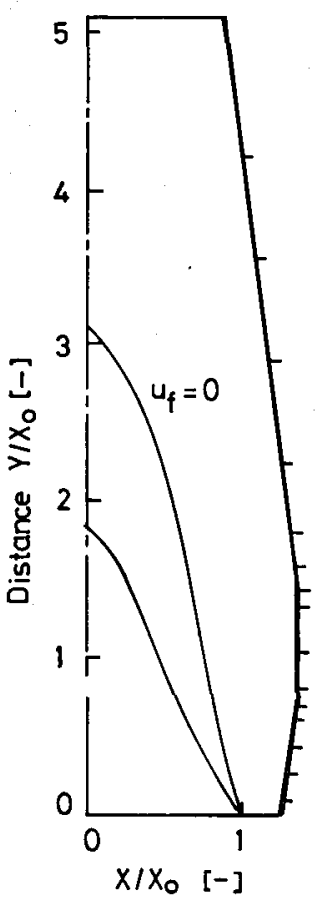

Fig. 6. Distribution of dynamic wall-pressure for sand.
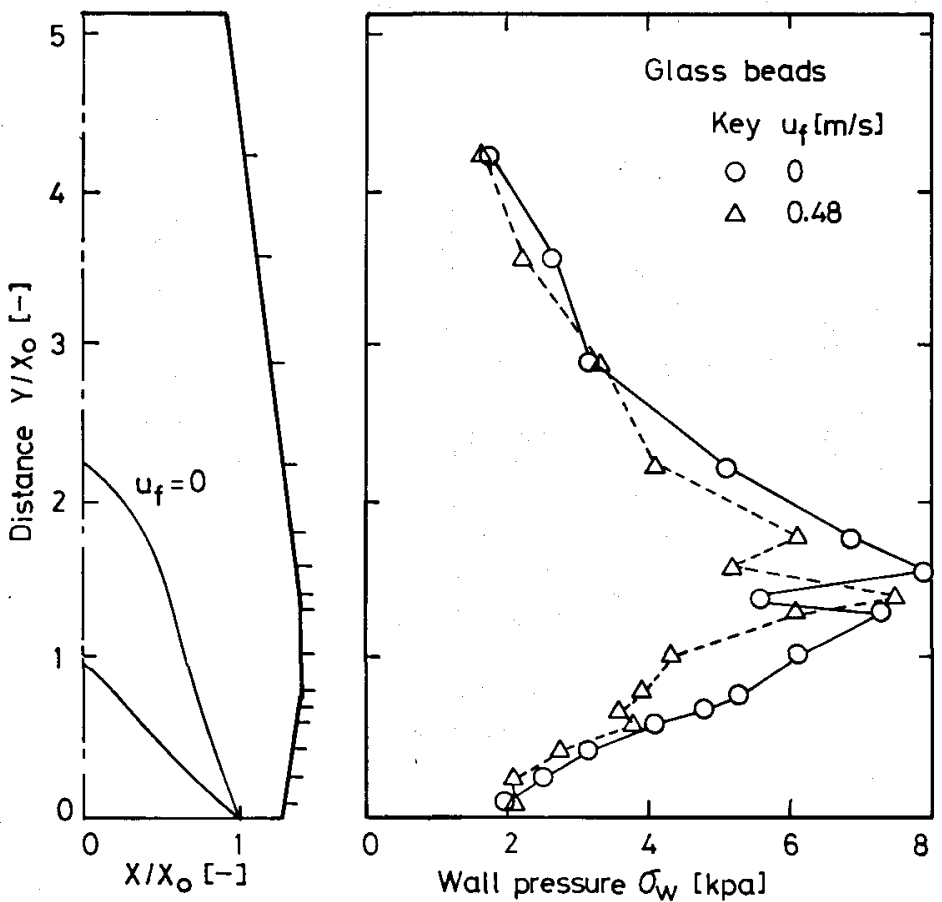

Fig. 7. Distribution of dynamic wall-pressure for glass beads. 
component because of the separation of flowing pass into the horizontal direction. This seems to be a reason for the increase in the wall pressure, as described by Shimizu et al. ${ }^{1 \prime}$ Such a stress condition can be assumed to be in the so-called passive state of Rankin.

\section{Galculation and Discussion}

\subsection{Theoretical Prediction of Characteristic Curves}

The pattern of the flow of particles is now considered in terms of plasticity theory. According to the theory, there are two different configurations associated with a discontinuity in the velocity ${ }^{12,13)}$ : one related to the characteristic curve of the stress, socalled "slip line" and one related to the characteristic curve of the velocity. The theory presented is concerned with two dimensional steady flow. Flowing material is assumed to be of homogeneous medium obeying a Coulomb-type failure criterion, $|\tau|=\tan \phi$. For the assumption to be applicable, the particle must be with a uniform size to prevent size segregation in the particles bed. Further its diameter $\left(d_{p}\right)$ must be sufficiently small compared with the characteristic length or size $(L)$ of the container to minimize the effect of the dimensions on the internal packing state. The relation, $d_{p} \leq L / 20$, suggested from the data after McGeary, ${ }^{14}$ may be utilized to estimate the effect. Provided this assumption is applicable, according to Sokolovski's technique, ${ }^{15)}$ the equation of slip line is given by the form

$$
d y / d x=\tan (\alpha \pm \eta)
$$

where, $x, y:$ the coordinates in horizontal and vertical directions, respectively

$$
\eta=\pi / 4-\phi / 2
$$

the variable $\alpha$ : angle between plane perpendicular to $x$ direction and major principal plane.

In the present paper, the upper side sign corresponds to a-slip line and the lower sign to b-slip line in Eq. (1). Fig. 8 shows the Mohr stress circle for the particles being deformed and Coulomb's yield condition (Y.L.). The characteristics related to slip lines are given by the points of contact, $\mathrm{A}$ and $\mathrm{B}$. The analytical solution for the loci of the slip line was derived by one of authors ${ }^{10)}$ as follows.

a-slip Line

$$
\begin{aligned}
& A\{(\alpha-\eta) \cos 2 \eta+\sin 2 \eta \ln |\cos (\alpha-\eta)|\}=-x+c_{1} \\
& \text {....................(2) } \\
& A\{(\alpha-\eta) \sin 2 \eta-\cos 2 \eta \ln |\cos (\alpha-\eta)|\}=y+c_{2}
\end{aligned}
$$

b-slip Line

$$
\begin{array}{r}
A\{(\alpha+\eta) \cos 2 \eta-\sin 2 \eta \ln |\cos (\alpha+\eta)|\}=-x+c_{3} \\
\ldots \ldots \ldots \ldots \ldots \ldots \ldots \ldots \ldots \ldots \ldots \\
A\{(\alpha+\eta) \sin 2 \eta+\cos 2 \eta \ln |\cos (\alpha+\eta)|\}=-y+c_{4}
\end{array}
$$

where, $c_{1} \sim c_{4}$ : integral constants

$$
A=2 \sigma_{m} \sin \phi / \gamma
$$

Applying the boundary values of $\alpha$ at both ends of the slip line gives the final formula for calculating the loci.

Fig. 9 shows the slip lines determined theoretically for the flow of sand by a method described below. The following were assumed in the calculation.

(1) The boundary of the stagnant region consists of a slip line and the slip line touches the central axis (i.e., axis of symmetry) at certain point $T$. The latter description is equivalent to assuming the point $T$ to be a singular point of stress.

(2) Stress condition of the flow field under the consideration is in the passive state of stress, based on the experimental results and discussion concerning Figs. 6 and 7.

Applying b-slip line for the boundary of the stagnant region, the locus can be evaluated from the following equations, derived from Eqs. (4) and (5).

$$
\begin{aligned}
& 1-x / x_{o}=f\left(\alpha, \alpha_{o}, \eta\right) / f\left(\alpha_{c}, \alpha_{o}, \eta\right) \\
& -y / x_{o}=g\left(\alpha, \alpha_{o}, \eta\right) / f\left(\alpha_{c}, \alpha_{o}, \eta\right)
\end{aligned}
$$

where the boundary condition for $\alpha$ was given as $\alpha=$ $\alpha_{o}$ at the edge of discharge port $\left(x=x_{0}, y=0\right)$ and $\alpha=$ $\alpha_{\varepsilon}$ at point $T$, and the functions $f(X, Y, \eta)$ and $g\left(X, Y, \gamma_{j}\right)$ are defined, with the variables $X$ and $Y$, by:

$$
\begin{aligned}
& f(X, Y, \eta)=(X-Y) \\
& \quad-\tan 2 \eta \ln |\cos (X+\eta) / \cos (Y+\eta)| \\
& g(X, Y, \eta)=(X-Y) \tan 2 \eta \\
& \quad+\ln |\cos (X+\eta) / \cos (Y+\eta)| \ldots \ldots \ldots \ldots
\end{aligned}
$$

The discharge angle $\phi_{d}$ is now defined, as shown in Fig. 9, by the slope of the stagnant region at the edge of discharge port. On Mohr stress circle shown in Fig. $8,2 \phi_{d}$ and $2 \alpha_{o}$ are given by the angles $\angle \mathrm{YOB}$ and $2 \pi-\angle \mathrm{QOX}$, respectively, and $2 \alpha_{c}=2 \pi-\angle \mathrm{QOB}$ since $\sigma_{x}$ at the point $T$ is given by the condition of

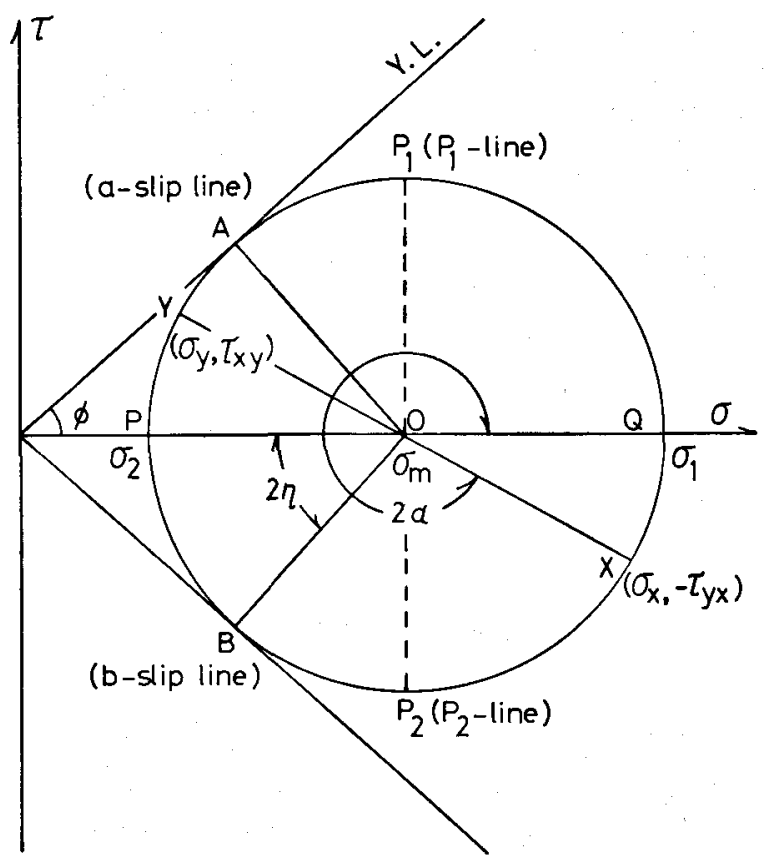

Fig. 8. Mohr stress circle and Coulomb's yield condition. 


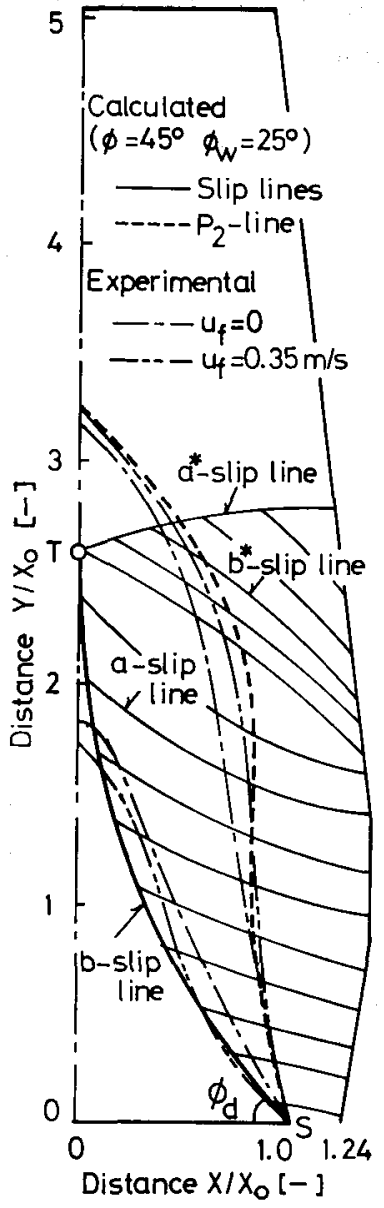

Fig. 9.

Comparison of calculated slip line and $P_{2}$-line for sand with experimental ones.

point B. Namely,

$$
\begin{aligned}
& \alpha_{o}=\pi-\phi_{d}+\eta \\
& \alpha_{c}=\pi / 2+\eta
\end{aligned}
$$

Assuming the condition $\phi_{d}=\phi$, the coordinates $(x, y)$ on b-slip line were calculated from Eqs. (7) and (8) corresponding to an arbitrary value of $\alpha$ chosen within $\alpha_{c}<\alpha<\alpha_{0}$. The position of point $T$ can be determined by substituting $\alpha_{c}$ for $\alpha$ in Eq. (8). The loci of another family consisting of a-slip lines, propagating from an arbitrary point $\left(x_{b}, y_{b}\right)$ on the b-slip line into the wall $\left(x=x_{R}\right)$, were determined from the following equations, derived from Eqs. (2) and (3).

$$
\begin{array}{r}
\left(x-x_{b}\right) /\left(x_{R}-x_{b}\right)=f\left(\alpha, \alpha_{b},-\eta\right) / f\left(\alpha_{R}, \alpha_{b},-\eta\right) \\
\ldots \ldots \ldots \ldots \ldots \ldots \ldots \\
\left(y-y_{b}\right) /\left(x_{R}-x_{b}\right)=g\left(\alpha, \alpha_{b},-\eta\right) / f\left(\alpha_{R}, \alpha_{b},-\eta\right)
\end{array}
$$

$\alpha_{b}$ is the value of $\alpha$ at the coordinate $\left(x_{b}, y_{b}\right)$ and the boundary values of $\alpha$ at the wall, $\alpha_{R}$ were evaluated from the conditions of points $R_{1}, R_{2}$ and $R_{3}$ on Mohr stress circle in Fig. 10. The points $R_{\mathbf{1}}, R_{\mathbf{2}}$ and $R_{3}$ correspond to the vertical, diverging and converging walls, respectively. The formulas obtained are

$$
2 \alpha_{R}=2 \pi-\phi_{w}-\sin ^{-1}\left(\sin \phi_{w} / \sin \phi\right) \pm 2 \theta
$$

where the upper side sign corresponds to the diverging wall, the lower sign to the converging wall and $\theta=0$ to the vertical wall. The calculated loci of a-slip lines are shown in Fig. 9.

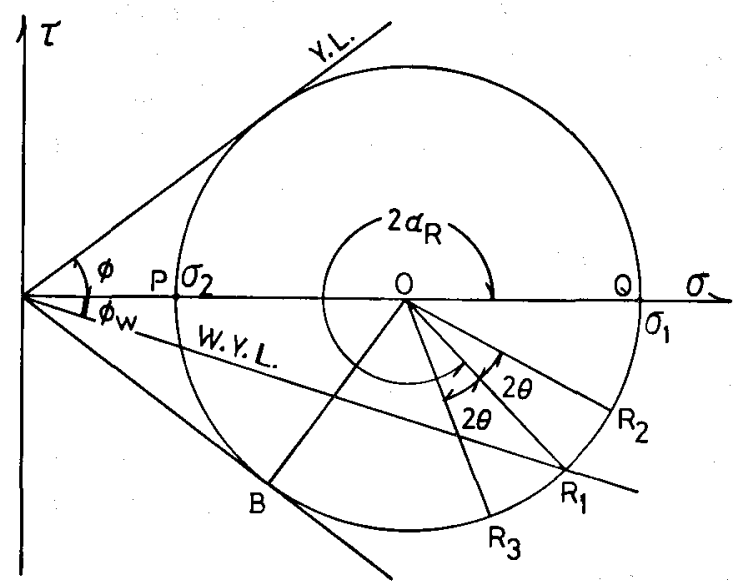

Fig. 10. Mohr stress circle representing wall conditions.

Another couple of a- and b-slip lines, propagating from the singular point of stress, $T$, is also shown in Fig. 9, denoted with $\mathrm{a}^{*}$ and $\mathrm{b}^{*}$. The derived formulas for the calculation are as follows.

$\mathrm{a}^{*}$-slip Line

$$
\begin{aligned}
& x / x_{R}=f\left(\alpha, \alpha_{c},-\eta\right) / f\left(\alpha_{R}, \alpha_{e},-\eta\right) \\
& \left(y-y_{c}\right) / x_{R}=g\left(\alpha, \alpha_{c},-\eta\right) / f\left(\alpha_{R}, \alpha_{c},-\eta\right)
\end{aligned}
$$

$\mathrm{b}^{*}$-slip Line

$$
\begin{aligned}
\left(x_{a}-x\right) /\left(x_{a}-x_{R}\right) & =f\left(\alpha, \alpha_{a}, \eta\right) / f\left(\alpha_{R}, \alpha_{a}, \eta\right) \\
\ldots \ldots \ldots \ldots \ldots \ldots \ldots & \ldots \ldots \ldots \ldots
\end{aligned}
$$

where the value of $\alpha$ at point $T, \alpha_{c}$, was evaluated from Eq. (20), assuming passive state of stress as stated before.

$$
\alpha_{\mathrm{c}}=\pi
$$

$\alpha_{a}$ denotes the value of $\alpha$ at an arbitrary coordinate $\left(x_{a}, y_{a}\right)$ on the $a^{*}$-slip line. As easily understood from the description above, the present method has the advantage of that the slip line can be estimated by simple algebraic calculation.

Since the theoretical characteristic curves of the velocity can be defined by points $P_{1}$ and $P_{2}$ in Fig. $8,{ }^{10,12)}$ they must be sloped at a constant angle from a- or b-slip line. Hence, the locus of the characteristic curve, propagating upward from point $S$, could be found by simple geometrical construction on the network of the slip lines. The determined curve, denoted as $P_{2}$-line, is shown in Fig. 9 in comparison with the experimental results. In the above method, the state of stress at the top of $P_{2}$-line was assumed to be still in the passive state. The theoretical curve corresponded fairly well to the boundary between the slow moving and fast moving regions. The result is a theoretical confirmation of the experimental fact that there was a discontinuous change in the velocity as well as the direction of flow on crossing the boundary. The boundary of the stagnant region could be also well represented by b-slip line, although there was some discrepancy between the experimental and 
calculated results near the axis of symmetry.

The theoretical results obtained for glass beads are compared with the experimental ones in Fig. 11. In this case, the discrepancy between the calculated slip line for the stagnant region and the experimentally obtained boundary was somewhat larger, while the theoretical characteristic curve of the velocity agreed well with the experimental one. The boundary of the stagnant region may be generally comprised by the envelope of slip lines, as shown by broken lines in Fig. 11.

The effect of friction properties of particles on the flow pattern can be estimated by comparing Fig. 3 with Fig. 5. More frictional material added larger sizes of the stagnant and slow moving regions.

\subsection{Flow Pattern in a Practical Furnace}

The experimental and theoretical results for the boundary of stagnant region in a three-dimensional blast furnace model, after Shimizu et al., ${ }^{11}$ are shown in Fig. 12 with the present theoretical curves described below. The friction properties of the particles used in their experiment, shown in Fig. 12, are close to those of sand in the present work, so that the comparison of their result with the present result related to sand is meaningful. Apparently, the height of the stagnant region formed in the three-dimcnsional model is lower than that formed in the two-dimensional model. The stress field set up in the bed of particles may depend on whether the apparatus is either two or three dimensional. Hence, the boundary condition of the singular point of stress on the

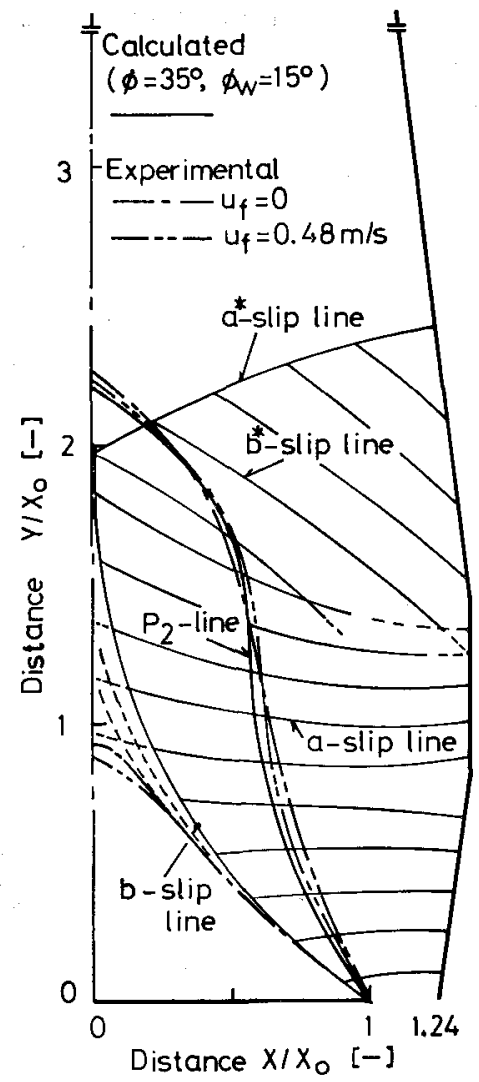

Fig. 11. Comparison of calculated slip lines and $P_{2}$-line for glass beads with experimental oncs. central axis may not be suitable for describing the stress field within blast furnace. It seems to be suitable to assume such a stress condition as satisfying Eq. (20) only for the top of the stagnant region in a practical furnace. Applying Eqs. (11) and (20) for $\alpha_{o}$ and $\alpha_{c}$, respectively, the following relations for evaluating the b-slip line were derived from Eqs. (4) and (5).

$$
\begin{aligned}
& -x / x_{o}=f\left(\alpha, \alpha_{c}, \eta\right) / f\left(\alpha_{c}, \alpha_{o}, \eta\right) \\
& -y / x_{o}=-g\left(\alpha, \alpha_{o}, \eta\right) / f\left(\alpha_{c}, \alpha_{o}, \eta\right)
\end{aligned}
$$

or

$$
-y / x=g\left(\alpha, \alpha_{o}, \eta\right) / f\left(\alpha, \alpha_{c}, \eta\right)
$$

The equation of $P_{2}$-line (the characteristic curve of the velocity) is given by the form. ${ }^{12,13)}$

$$
d y / d x=\tan (\alpha-\pi / 4)
$$

Eqs. (1) and (24) can be numerically integrated with the aid of Eq. (23). This provides the numerical calculation of the characteristic curves, which is a different method from that with the use of Eqs. (7) to (20). The loci of the b-slip line and $P_{2}$-line propagating upward from the edge of the outlet, determined for coke particles, ${ }^{1)}$ are shown in Fig. 12. The experimental data as well as the theoretical result after Shimizu et al. $^{\text {1) }}$ for the boundary of the stagnant region were in the range between the two present theoretical curves. However, the experimental confirmation about that the flow pattern was measured after the flow attained the steady state flow, was not

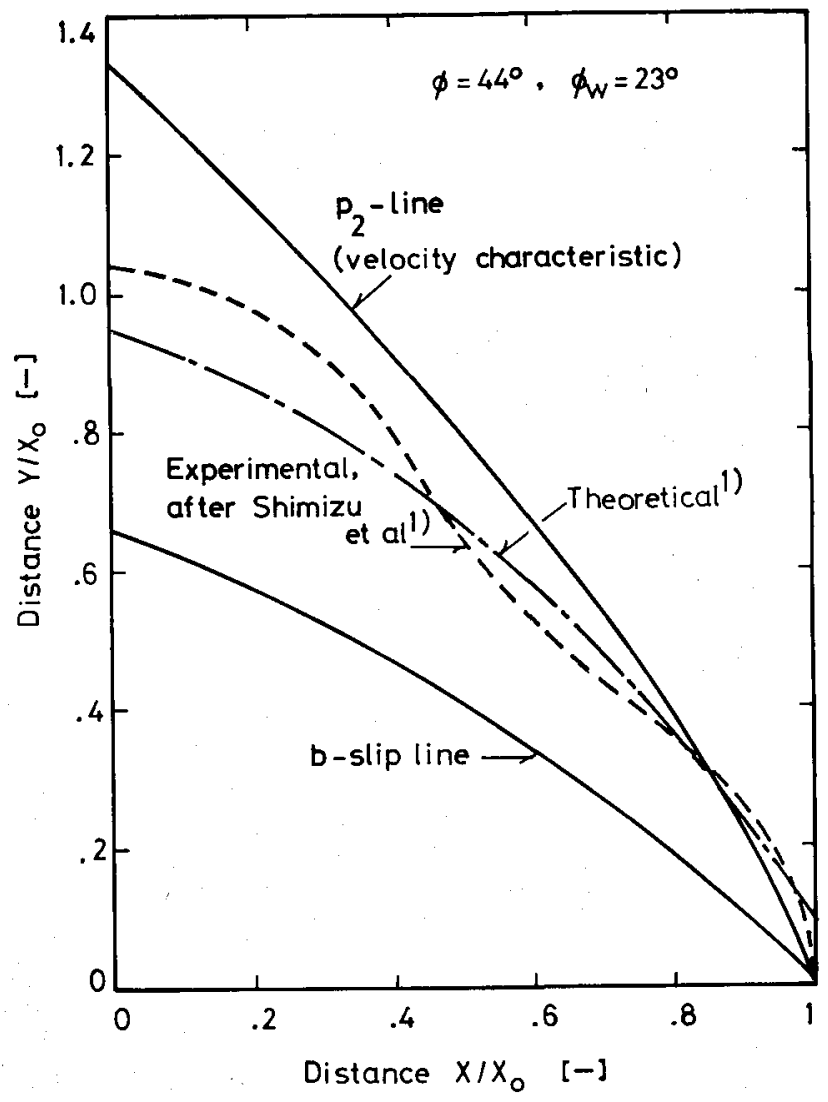

Fig. 12. Comparison of calculated slip lines and $P_{2}$-line for a blast furnace with experimental one. 
given and further, any distinction between the slip line and the velocity characteristic line was not done. The discrepancy between the present and their theoretical results is mainly originated from the assumption applied for the stress field: $\sigma_{m}=$ constant $^{10)}$ in this work and $\tau \propto x^{11}$. A careful measurement and detailed analysis of the flow pattern of particles in a three dimensional model of the blast furnace are needed to confirm the applicability of the previous theories ${ }^{1,8)}$ as well as the present theory.

\section{Conclusion}

Using a two-dimensional cold model of iron making blast furnace, the flow pattern and dynamic wallpressure of particles were carefully measured in order to study the motion of the particles in the blast furnace. A detailed analysis of the flow pattern revealed the fact that the flow field could be devided into three characteristic regions: stagnant, slow moving and fast moving regions. The existence of characteristic points at which the velocity and direction of flow of the particles changed discontinuously was revealed experimentally, and the boundary between the slow moving and fast moving regions consisted of these characteristic points. An approximate calculus of estimating the boundary between each of the characteristic regions was described on the basis of plasticity theory. The boundary of the stagnant region could be represented by the theoretical characteristic curve of the stress, so-called slip line. The boundary between two regions with different velocity characteristics corresponded very well to the theoretical characteristic curve of the velocity. Finally, a flow pattern to be set up in a practical blast furnace was simulated. The result did not always agree with the reported experimental data. It was concluded that a more careful measurement of the flow pattern in a three dimensional model of the blast furnace is required for the confirmation of the present and previous theories.

\section{Nomenclature}

$B:$ direction of flow of particles $\left({ }^{\circ}\right)$

$d_{p}$ : diameter of particle $(\mathrm{m})$

$t:$ time after the start of discharge ( $\mathrm{min}$ )

$U_{x}, U_{y}:$ horizontal and vertical velocities, respectively $(\mathrm{m} / \mathrm{s})$

$x$ : horizontal distance measured from central axis $(\mathrm{cm})$

$x_{B}, x_{M}$ : horizontal distances defined in Fig. 4, re- spectively $(\mathrm{cm})$

$x_{0}$ : distance from central axis to outlet $(\mathrm{cm})$

$x_{R}$ : distance from central axis to wall $(\mathrm{cm})$

$y$ : vertical distance measured downward $(\mathrm{cm})$

$\alpha$ : angle between plane perpendicular to $x$ direction and major principal plane $\left({ }^{\circ}\right)$

$\alpha_{c}, \alpha_{R}$ : values of $\alpha$ at $x=0$ and $x=x_{R}$, respectively $\left({ }^{\circ}\right)$

$\gamma:$ bulk density of particles $\left(\mathrm{kg} / \mathrm{m}^{3}\right)$

$\varepsilon$ : void fraction $(-)$

$\eta=\pi / 4-\phi / 2\left(^{\circ}\right)$

$\theta$ : angle of bosh or shaft $\left(^{\circ}\right)$

$\rho_{p}:$ density of particle $\left(\mathrm{kg} / \mathrm{m}^{3}\right)$

$\sigma_{1}, \sigma_{2}$ : major and minor principal stresses, respectively $(\mathrm{Pa})$

$\sigma_{m}:$ mean principal stress $(\mathrm{Pa})$

$\sigma_{w}: \quad$ wall pressure $(\mathrm{Pa})$

$\tau: \quad$ shear stress $(\mathrm{Pa})$

$\dot{\phi}:$ angle of internal friction $\left({ }^{\circ}\right)$

$\phi_{d}:$ angle of discharge $\left({ }^{\circ}\right)$

$\phi_{w}$ : angle of wall friction $\left({ }^{\circ}\right)$

\section{REFERENCES}

1) M. Shimizu, A. Yamaguchi, S. Inaba and K. Narita: Tetsu-to-Hagané, 68 (1982), 936.

2) N. Miyasaka, M, Suga, Y. Hara and S. Kondo: Tetsu-toHagané, 18 (1972), 58.

3) K. Isobe, M. Kuwabara and I. Muchi: Tetsu-to-Hagané, 67 (1981), S53; Trans. Iron Steel Inst. Jpn., 21 (1981), B386.

4) L. Z. Khodak and Yu. I. Borisov: Powder Technol., 4 (1970/ 71), 535 .

5) K. Kanbara, T. Hagiwara, A. Shigemi, S. Kondo, Y. Kanayama, K. Wakabayashi and N. Hiramoto: Tetsu-to-Hagané, $62(1976), 535$.

6) K. Narita, S. Inaba, M. Shimizu, T. Kobayashi, K. Okimoto and A. Yamaguchi: Tetsu-to-Hagané, 66 (1980), 459.

7) M. Shimizu, Y. Kimura, M. Isobe, C. Che and S. Inaba: Tetsu-to-Hagané, 73 (1987), 1996.

8) H. Nishio, W. Wenzel and H. W. Gudenau: Stahl u. Eisen, 97 (1977), 867.

9) H. Nishio, T. Ariyama, Y. Tanba, A. Yamaguchi and K. Wakimoto: Tetsu-to-Hagané, 65 (1979), 601.

10) H. Takahashi and H. Yanai: Kagaku Kogaku (Chem. Eng. Jpn.), 38 (1974), 746.

11) H. Takahashi, E. Obata and T. Takeuchi: Kagaku Kogaku Ronbunshu, 13 (1987), 340.

12) Y. Yamada: Sosei Rikigaku (Plasticity Mechanics), Nikkan Kogyo Shinbunsha, Tokyo, (1982), 182, 206.

13) J. R. Johanson: J. Appl. Mech., Trans. ASME, (1964), Sept., 499.

14) R. K. McGeary: J. Am. Ceramic Soc., 44 (1961), 513.

15) K. Hoshi and K. Sato: Statics of Soil Media, Ohmu-sha, Tokyo, (1964), 22. 